\title{
Reentanglement Kinetics in Sheared Polybutadiene Solutions
}

\author{
C. G. Robertson, ${ }^{*},{ }^{\dagger}$ S. Warren, ${ }^{\dagger}$ D. J. Plazek, ${ }^{\ddagger}$ and C. M. Roland ${ }^{*, \S}$ \\ Bridgestone Americas Center for Research and Technology, 1200 Firestone Parkway, \\ Akron, Ohio 44317-0001; Department of Materials Science and Engineering, University of Pittsburgh, \\ Pittsburgh, Pennsylvania 15261; and Naval Research Laboratory, Chemistry Division, Code 6120, \\ Washington, D.C. 20375-5342
}

Received September 9, 2004; Revised Manuscript Received September 30, 2004

\begin{abstract}
Interrupted shear measurements were used to follow the reentanglement kinetics and associated molecular weight dependence for solutions of near-monodisperse polybutadiene (PB). The timedependent recovery of the overshoot in the shear stress was measured for PB having weight-average molecular weights $\left(M_{\mathrm{w}}\right)$ equal to $61,90,107$, and $167 \mathrm{~kg} / \mathrm{mol}$, corresponding to a range of 17-47 entanglements per chain in solution. The times for recovery of the stress overshoot were 1.5 decades longer (30 times greater) than the corresponding linear viscoelastic relaxation times, the latter determined from dynamic shear data or from stress relaxation following cessation of flow. Notwithstanding the differing time scales, the $M_{\mathrm{w}}$ dependences for entanglement recovery and linear viscoelastic relaxation were equivalent (power law exponent $\sim 3.4$ ).
\end{abstract}

\section{Introduction}

Deformation and flow of polymers are central to most processing operations (calendering, extrusion, molding, etc.), and thus much effort has been expended over the years in understanding polymer rheology. For long, linear polymers, interpretations of the behavior are almost invariably based on reptation, whose central tenet is that longitudinal motions are favored due to entanglement constraints on motion transverse to the chain. Many experimental results can be satisfactorily described by tube models based on this idea ${ }^{1-3}$ especially when additional relaxation mechanisms are included, such as contour length fluctuations (CLF) of the reptating chain ${ }^{1,2,4}$ and constraint release (CR) from motion of neighboring chains. ${ }^{1,2,5}$ One controversy concerns molecular weight dependences. The viscosity, $\eta$, and terminal relaxation time, $\tau$, of entangled polymers are predicted to vary as $M^{3}$, where $M$ is molecular weight; however, a large body of data indicates $\eta \propto \tau \propto M^{3.4 \pm 0.2}$. This stronger $M$ dependence is generally considered to be a consequence of CLF and CR mechanisms operative at lower molecular weights, such that the predicted $M^{3}$ would be recovered for sufficiently long chains $(M>$ $200 M_{\mathrm{e}}$, where $M_{\mathrm{e}}$ is the entanglement molecular weight). ${ }^{6}$ Reptation with CLF is predicted to yield a 3.4 exponent, ${ }^{7}$ while generally $\mathrm{CR}$ does not alter the 3.0 exponent predicted for simple reptation..$^{5,8,9}$ On the other hand, CR via double reptation was found to yield an $M$ dependence of $\eta$ consistent with experimental data. ${ }^{10}$ The tracer and self-diffusion constants vary as $M^{-2}$, which is consistent with a cubic-power law for the viscosity and $\tau$. Evidently, asymptotic behavior is attained at lower $M$ for diffusion than for relaxation, due perhaps to an absence of CLF effects on the diffusion ${ }^{11-13}$ (although see ref 7).

When polymers are subjected to flow, CR and CLF mechanisms become particularly important for describ-

\footnotetext{
Bridgestone Americas Center for Research and Technology. University of Pittsburgh.

$\S$ Naval Research Laboratory.

* Corresponding authors. E-mail robertsonchristopher@bfusa.com and roland@nrl.navy.mil.
}

ing the viscoelastic behavior. ${ }^{2,14-18}$ Flow at high rates reduces the entanglements on a chain ${ }^{19}$ by a process referred to as convective CR. ${ }^{2,20}$ The disentanglement of polymers subjected to steady shearing is wellestablished experimentally. ${ }^{21-27}$ In a steady shearing experiment, the loss of entanglements leads to a maximum in the stress ("stress overshoot") at high shear rates. Stress overshoots are also observed in dilute polymer solution, ${ }^{28}$ as well as melts of low molecular weight (unentangled) polymers, ${ }^{29,30}$ due to molecular orientation. However, it is the larger overshoots in entangled polymers that are of interest herein. At strains beyond the maximum, the stress assumes a constant steady-state value, reflecting an equilibrium between the rates of flow-induced chain disentanglement and reentanglement via thermally activated motion. Upon cessation of the shearing, the stress decays in a rather complicated fashion, with the long-term behavior exhibiting the same $M^{3.4}$ dependence as the viscosity. ${ }^{31}$ After the flow has stopped, lost entanglements are recovered, which upon resumption of the flow leads to a time-dependent recovery of the stress overshoot. ${ }^{32-36}$ The maximum overshoot occurs at a shear strain in the range of $2-3$, independent of the duration of the rest period. ${ }^{35,37}$ Complete recovery of the overshoot (that is, complete reentanglement) requires substantially longer times than the time for stress relaxation after cessation of the shearing. ${ }^{32,34}$

In this work, we measure stress overshoot and recovery of entangled polybutadiene solutions. Our specific interest is determination of the time for recovery of the equilibrium entanglement density, as reflected in the maximum in the stress overshoot, and its dependence on molecular weight. While the molecular weight dependence of the linear viscoelastic properties of entangled polymers is well-established, to our knowledge this is the first determination of the molecular weight dependence of the reentanglement time following nonlinear flow.

We study concentrated solutions of 1,4-polybutadiene (PB). The use of solutions minimizes slip and other problems with achieving a uniform flow. Of course, dilution decreases the entanglement concentration; 
Table 1. Polybutadienes

\begin{tabular}{|c|c|c|c|c|c|c|}
\hline polymer & $M_{\mathrm{w}}(\mathrm{kg} / \mathrm{mol})$ & $M_{\mathrm{w}} / M_{\mathrm{n}}$ & $M_{z} / M_{\mathrm{w}}$ & $\%$ 1,2 (vinyl) & $\%$ trans $-1,4$ & $\%$ cis $-1,4$ \\
\hline PB61K & 61.0 & 1.03 & 1.04 & 9.0 & 49.9 & 41.1 \\
\hline PB90K & 89.6 & 1.04 & 1.04 & 8.8 & 53.5 & 37.7 \\
\hline PB107K & 107.4 & 1.03 & 1.05 & 9.2 & 53.7 & 37.1 \\
\hline PB167K & 166.9 & 1.04 & 1.04 & 9.2 & 50.1 & 40.7 \\
\hline
\end{tabular}

however, PB has a very small $M_{\mathrm{e}}=1850 \mathrm{~g} / \mathrm{mol},{ }^{6}$ so for the molecular weights used herein, the chains are wellentangled even for polymer concentrations $\sim 0.5$.

\section{Experimental Section}

The polybutadienes (Table 1 ) were synthesized by anionic polymerization at $55-60{ }^{\circ} \mathrm{C}$ in hexane, using $n$-butyllithium as an initiator. To obtain rapid termination, the reaction solution was transferred from the reactor directly into an excess of 2-propanol. The coagulant contained butylated hydroxyltoluene as a stabilizer, yielding ca. $1000 \mathrm{ppm}$ in the dried polymer. The chemical structure of the polybutadienes was determined using ${ }^{1} \mathrm{H}$ and ${ }^{13} \mathrm{C}$ NMR and absolute molecular weights by GPC in combination with the intrinsic viscosities (universal calibration method). The glass transition temperatures were equal for all samples, $T_{\mathrm{g}}=-92.3 \pm 0.4{ }^{\circ} \mathrm{C}$, as measured by differential scanning calorimetry at a heating rate of $5{ }^{\circ} \mathrm{C} / \mathrm{min}$.

Solutions of the polybutadienes in aromatic oil (Sundex 790 from Sun Oil Co.) were prepared by codissolution in hexane, followed by evaporation of the hexane in a vacuum oven. The polymer volume fraction was $\phi=0.52$ (equal to $0.49 \mathrm{~g}$ of $\mathrm{PB}$ per $\mathrm{mL}$ of solution).

Isothermal oscillatory shear measurements of the linear dynamic properties were carried out with a Rheometrics ARES instrument, for frequencies in the range $0.001 \leq \omega(\mathrm{rad} / \mathrm{s}) \leq$ $100 \mathrm{rad} / \mathrm{s}$. A parallel plate geometry was used, with sample diameters equal to 15,25 , or $40 \mathrm{~mm}$ depending on temperature, and sample thicknesses in the range from 1.6 to $3.5 \mathrm{~mm}$. Thermal stability of the samples at the highest temperature of $75^{\circ} \mathrm{C}$ was verified by replicate testing at low frequency. Transient and steady-state shear measurements of shear stress and first normal stress difference were also conducted at $25{ }^{\circ} \mathrm{C}$ using the ARES, with a cone and plate geometry. A diameter of $25 \mathrm{~mm}$ (cone angle $=0.04 \mathrm{rad}$ ) was used for low shear rate determinations of the zero-shear viscosities, while for steady-state shearing at higher rates and the shear recovery testing, a $15 \mathrm{~mm}$ diameter (cone angle $=0.1 \mathrm{rad}$ ) was used. To ensure good adhesion of the sample, the cone and plate were lightly abraded with 200 grit sandpaper and cleaned with solvent. To further enhance adhesion, a small amount of $\mathrm{PB}-$ oil solution diluted with hexane was painted on the fixtures. After evaporation of the hexane, additional sample was added to fill the gap. Annealing for $1 \mathrm{~h}$ at $50{ }^{\circ} \mathrm{C}$ was performed prior to measurements at $25^{\circ} \mathrm{C}$. The startup transient experiments employed respective shear rates equal to $70 \mathrm{~s}^{-1}$ (PB61K), $20 \mathrm{~s}^{-1}$ (PB90K), $10 \mathrm{~s}^{-1}$ (PB107K), and 2.5 $\mathrm{s}^{-1}(\mathrm{~PB} 167 \mathrm{~K})$; these were chosen on the basis of the magnitudes of the terminal relaxation time determined for the samples (as discussed below). While it is generally held that the use of cone and plate geometry provides a uniform deformation rate across the gap, we note that one recent work suggests that disentanglement does not take place uniformly across the sample during shear. ${ }^{38}$ However, the stresses herein were well below the level, relative to the plateau modulus, at which the putative inhomogeneous flow transpires.

\section{Results and Discussion}

In Figure 1 are displayed master curves of the storage, $G^{\prime}$, and loss, $G^{\prime \prime}$, moduli for the four PB solutions. To construct the master curves, horizontal shift factors, $a_{\mathrm{T}}$, were determined by superposition of the loss tangent ( $\tan \delta$ ) data, with small vertical shift factors, $b_{\mathrm{T}}$, then determined by superposition of the modulus functions. The high-frequency plateaus in the storage modulus for the solutions in Figure 1 superimpose on the corresponding plateau zone for the neat samples (not shown), by a vertical shift equal to $3.8 \pm$ 0.1 . This corresponds to a quadratic dependence of the plateau modulus on polymer concentration, in accord with literature results, $G_{N}^{0} \propto \phi^{a}(2 \leq a \leq 2.3) .{ }^{39}$ This means that the entanglement molecular weight will vary as $M_{\mathrm{e}} \propto \phi^{1-a}$. We calculate that $M_{\mathrm{e}}$ increases from $1850 \mathrm{~g} / \mathrm{mol}$, the value for neat $\mathrm{PB},{ }^{6}$ to $3560 \mathrm{~g} / \mathrm{mol}$ by dilution with the oil. Thus, the solutions have from 17 to 47 entanglements per chain; that is, they are sufficiently entangled to exhibit the high molecular weight limiting behavior for their rheological properties.

In Figure 2 the terminal values of the dynamic viscosity, $\eta^{\prime}{ }_{0}=\lim _{\omega \rightarrow 0} G^{\prime \prime} / \omega$, for both the neat PB and the solutions are plotted vs molecular weight. The expected power-law behavior with an exponent of 3.4 is observed. Also shown are some published results ${ }^{6}$ for neat $\mathrm{PB}$, which fall in line with the data measured herein. The zero-shear-rate-limiting values of the steadystate shear viscosity, $\eta_{0}=\lim _{\dot{\gamma} \rightarrow 0} \eta_{\mathrm{ss}}(\dot{\gamma})$, are included in Figure 2. They are equal to the dynamic viscosities within the experimental error for such measurements.

In fact, this agreement extends to the highest rates at which steady-state data could be obtained, as shown in Figure 3 for the PB solutions. Such correspondence between dynamic and steady-state viscosities is referred to as the Cox-Merz rule. ${ }^{40-43}$ Another empiricism is the Gleissle relation, according to which the steady-state viscosity is equal to the transient viscosity measured during approach to steady state at the limiting shear rate, ${ }^{44} \eta_{\mathrm{ss}}(\dot{\gamma})=\left.\lim _{\dot{\gamma} \rightarrow 0} \eta^{+}(t, \dot{\gamma})\right|_{t^{-1}=\dot{\gamma}}$. The subscript denotes that the startup viscosity is taken at a shearing time equal to the reciprocal of the corresponding shear rate. The Gleissle relation has been shown to work well for a number of entangled polymers. ${ }^{45-48}$ As seen in Figure 3 , the shear viscosity deduced from the transient stress agrees with the dynamic viscosity to within $20 \%$, over

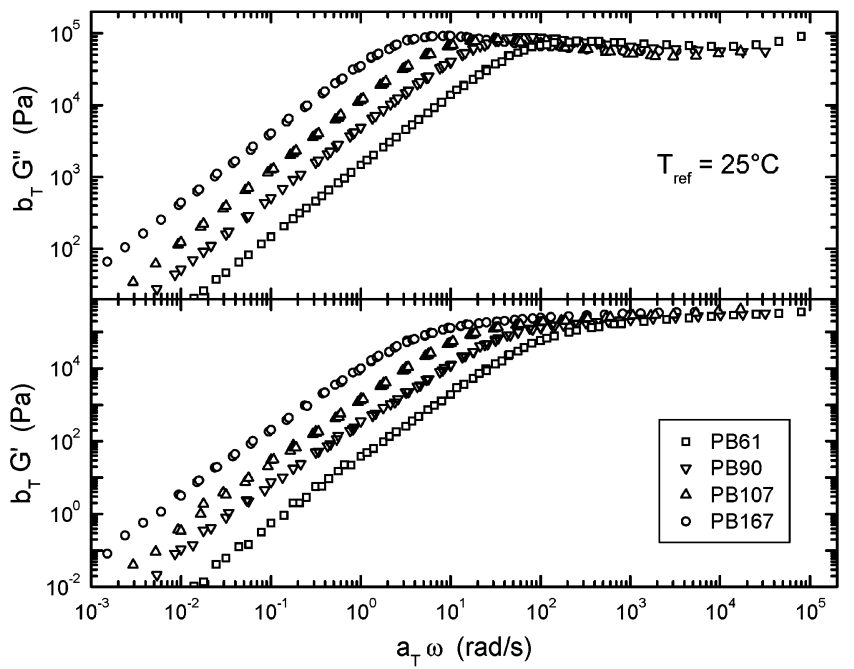

Figure 1. Master curves of the storage and loss moduli of the four $\mathrm{PB}$ solutions at a common reference temperature of $25{ }^{\circ} \mathrm{C}$. 


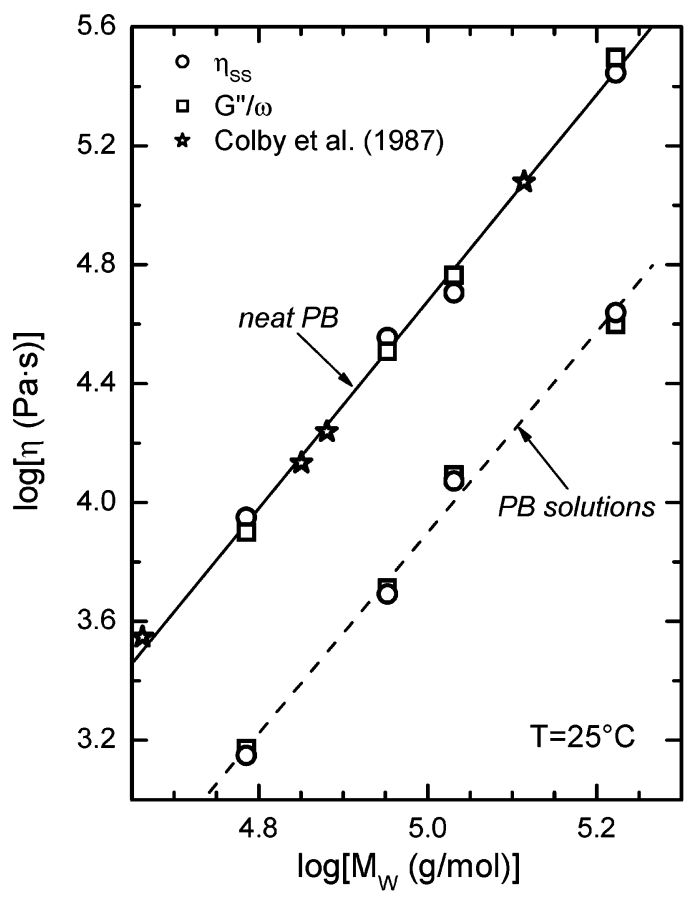

Figure 2. Molecular weight dependence of the terminal viscosities measured for the PB neat and in concentrated $(\phi$ $=0.52$ ) solutions. The lines are fits, yielding $3.4 \pm 0.1$ for the exponent. Included are the data of Colby et al. ${ }^{6}$ for neat polybutadienes having similar microstructure.

a range encompassing up to a 20 -fold change in viscosity.

We can extract the steady-state recoverable compliance, $J_{S}^{0}$, from the shear data, as $J_{S}^{0}=\Psi_{0} / 2 \eta_{0}^{2}$, where $\Psi_{0}$ is the ratio of the first normal stress difference, $N_{1}$, and the square of the shear rate, measured at low shear rate. We obtain $J_{S}^{0}=19 \pm 1 \mathrm{MPa}^{-1}$, independent of molecular weight. Using this value of $J_{S}^{0}$ and the zeroshear viscosities, we calculate the terminal relaxation times $^{1}$

$$
\tau_{\eta}=\frac{15}{\pi^{2}} \eta_{0} J_{S}^{0}
$$

These terminal relaxation times vary with molecular weight according to the 3.4 power (Figure 4), consistent with past studies. ${ }^{6,49}$ Compared to the steady-state evaluation of $J_{S}^{0}$, the low-frequency dynamic data are less reliable in obtaining $J_{S}^{0}$ as $\lim _{\omega \rightarrow 0} J^{\prime}(\omega)$. Nonetheless, we estimated relaxation times from the dynamic data using eq 1 . The values of $\log \left(\tau_{\eta}\right)$ determined in this manner are $-1.19,-0.61,-0.33$, and 0.09 for PB61K, PB90K, PB107K, and PB167K, respectively, in acceptable agreement with the steady-state results given in Table 2.

Shear experiments were carried out at a rate equal to thrice the reciprocal of the terminal relaxation time, $\dot{\gamma}=3 / \tau_{\eta}$. The combination of high rate and large strains causes chain disentanglement and a consequent overshoot in both the shear and normal stresses. In Figure 5, representative results are shown for PB90K as a function of shearing time. For all four solutions, the maximum in the shear stress had a magnitude equal to $1.32 \pm 0.02$ times the steady-state stress for fully equilibrated samples. This peak transpired at a molecular-weight-independent shear strain $=2.76 \pm 0.26$. Although not readily apparent on the linear time axis

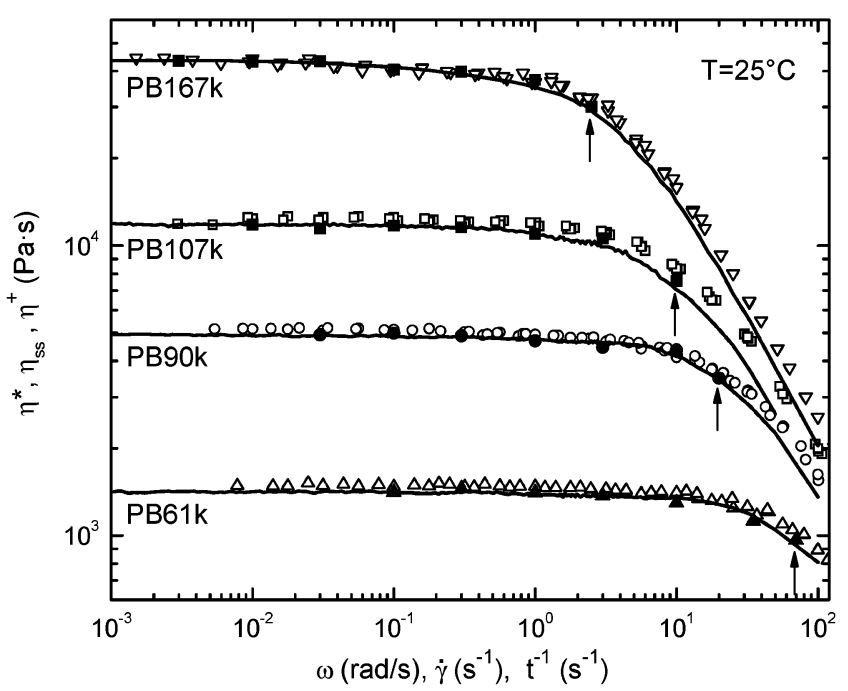

Figure 3. Steady-state shear viscosities (solid symbols), dynamic shear viscosities (open symbols), and the transient viscosities (line), the latter determined from the lowest shear rates using the Gleissle relation and plotted vs the reciprocal of the shear time. The arrows indicate the shear rates at which the overshoot recovery measurements were made.

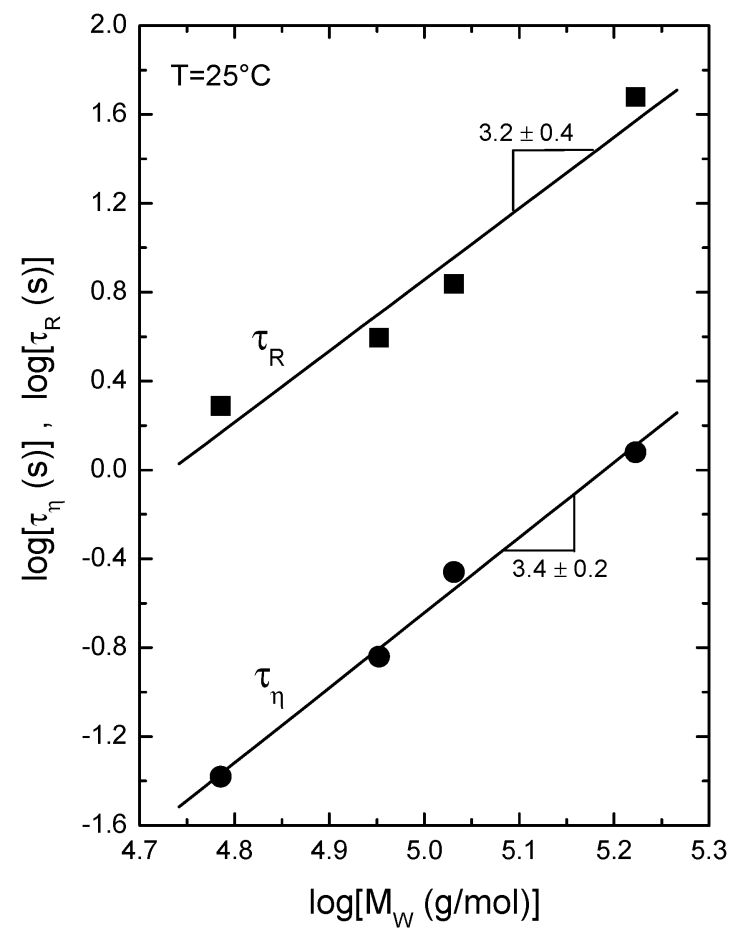

Figure 4. Molecular weight dependence of the terminal relaxation time $(\bullet)$ and the time for complete recovery of the startup transient in the shear stress (ם).

Table 2. Rheological Results for Polybutadiene Solutions

\begin{tabular}{lclcc}
\hline polymer & $M_{\mathrm{w}} / M_{\mathrm{e}}$ & $\log \eta_{0}(\mathrm{~Pa} \mathrm{~s})$ & $\log \tau_{\eta}(\mathrm{s})$ & \multicolumn{1}{c}{$\log \tau_{\mathrm{R}}(\mathrm{s})$} \\
\hline PB61K & 17 & $3.16 \pm 0.01$ & $-1.38 \pm 0.02$ & $0.29 \pm 0.030$ \\
PB90K & 25 & $3.70 \pm 0.01$ & $-0.84 \pm 0.03$ & $0.59 \pm 0.019$ \\
PB107K & 30 & $4.08 \pm 0.01$ & $-0.46 \pm 0.03$ & $0.84 \pm 0.025$ \\
PB167K & 47 & $4.62 \pm 0.015$ & $0.08 \pm 0.03$ & $1.68 \pm 0.017$
\end{tabular}

in Figure 5, the first normal stress difference reached a maximum at a larger strain than the peak in the shear stress. This strain difference, which decreased weakly with molecular weight, was equal to a factor of 2.2 on average, in agreement with the results of Menezes and Graessley, ${ }^{35}$ who reported that the $N_{1}$ maximum oc- 


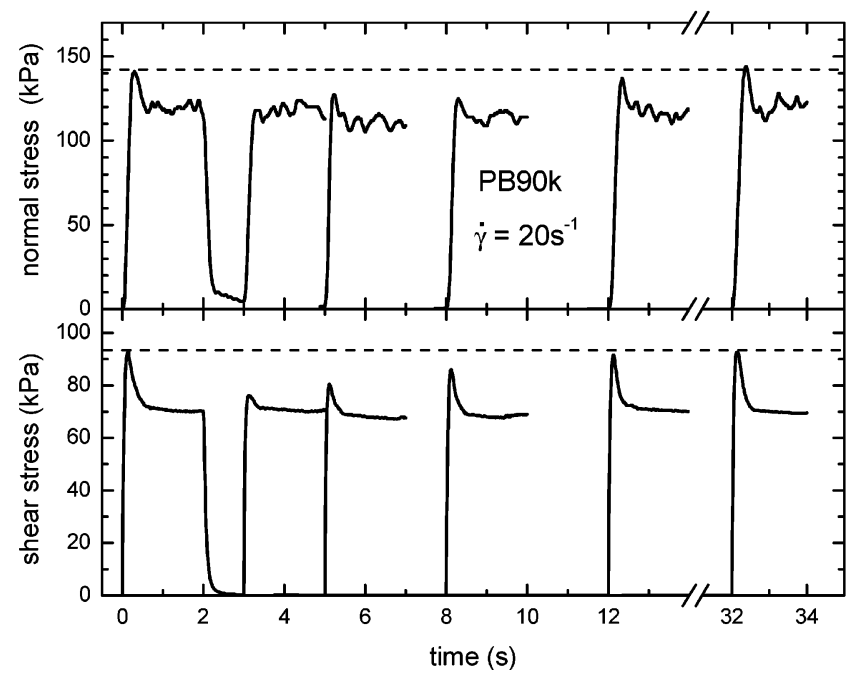

Figure 5. Transient shear stress (lower panel) and first normal stress difference (upper panel) for PB90K at $25^{\circ} \mathrm{C}$ and a shear rate equal to $20 \mathrm{~s}^{-1}$. Left-most curves are for shearing from full equilibrium (rest time $=1000 \mathrm{~s}$ ) followed by stress relaxation while unsheared. The remaining curves are for resumption of the shearing after recovery periods of $1,3,6$, 10 , and $30 \mathrm{~s}$ (from left to right).

curred at a strain that was $2.3 \pm 0.2$ times the strain for the shear stress maximum.

To study the reentanglement process, interrupted shear flow experiments were carried out. Following attainment of steady state, the flow was stopped. During the rest period, the stress relaxed, with a time dependence related to $J_{S}^{0}$ and the preceding shear rate. ${ }^{34}$ After a time equal to the $\tau_{\eta}$ determined from the linear viscoelastic measurements, the stress had decayed by 1 decade.

Following the zero-shear rest period and complete relaxation of the stress, the shearing was resumed. The startup transient peaks in $\eta$ and $N_{1}$ reappeared, with amplitudes dependent on the duration of the rest time (Figure 5). The scatter in the normal stress makes accurate determination of the kinetics difficult. However, this is not the case for the shear stress, and in Figure 6, the magnitude of its overshoot peak is plotted as a function of recovery time for the four PB solutions. Following Stratton and Butcher, ${ }^{34}$ the data can be satisfactorily fit to the recovery function

$$
\eta_{\max }^{+}=\eta_{\mathrm{ss}}+A\left(1-\mathrm{e}^{-t / \tau_{\mathrm{R}}}\right)
$$

where $\eta_{\mathrm{ss}}$ is the steady-state viscosity, $A$ is a constant, and $\tau_{\mathrm{R}}$ is a characteristic time for recovery of the overshoot in the transient viscosity, $\eta_{\text {max }}^{+}$. The obtained $\tau_{\mathrm{R}}$ values, representing the times required for reentanglement of the sheared polymers, are displayed in Figure 4 as a function of PB molecular weight. We find that the reentanglement process has a molecular weight dependence, $\tau_{\mathrm{R}} \sim M_{\mathrm{w}}{ }^{3.2 \pm 0.4}$, that is the same within the error as that found for the linear viscoelastic behavior of the fully entangled PB (cf. $\tau_{\eta}$ in Figure 4). Notwithstanding this equivalence, the reentanglement requires substantially longer times than the corresponding linear viscoelastic relaxation times; i.e., $\tau_{\mathrm{R}}$ is ca. 1.5 decades greater than $\tau_{\eta}\left(\tau_{\mathrm{R}} \approx 30 \tau_{\eta}\right)$.

The observation of $\tau_{\mathrm{R}} \gg \tau_{\eta}$ is not anticipated by theoretical models, nor can integral constitutive equations accurately describe these reentanglement kinetics. ${ }^{50,51}$ The unresolved physics leading to $\tau_{\mathrm{R}} \gg \tau_{\eta}$ may

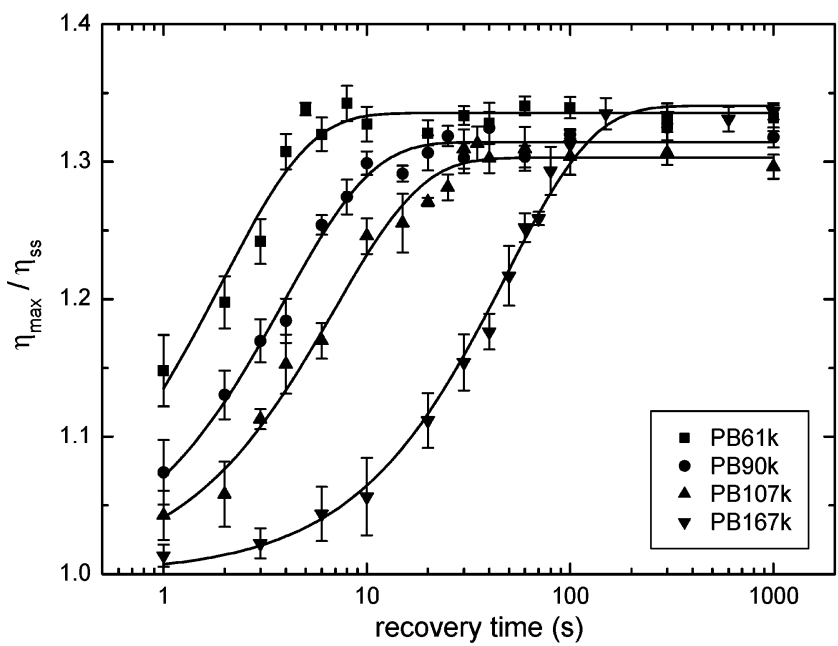

Figure 6. Magnitude of the overshoot in the transient viscosity normalized by the steady-state viscosity as a function of the rest time in between shearing. Data represent the average of three measurements. Lines are fits to an exponential growth function (eq 2).

be responsible for other unexplained nonlinear rheological phenomena such as the anomalously slow growth of extrudate swell relative to the time scale for terminal relaxation..$^{52}$ Our results are consistent with the limited number of previous studies reporting on the time dependence of the stress overshoot recovery. Stress relaxation following cessation of flow was approximately 1 decade faster than $\tau_{\mathrm{R}}$ for a $3 \%$ entangled polyisobutylene solution. ${ }^{34}$ For a $0.07 \mathrm{~g} / \mathrm{mL}$ solution of very high molecular weight polybutadiene in oil, the time to fully recover the stress overshoot peak was 1.3 decades longer than $\tau_{\eta} \cdot{ }^{35}$ Commercial polyethylene melts exhibited $\tau_{\mathrm{R}}$ that were about 2 decades greater than $\tau_{\eta}{ }^{32}$ None of these earlier works investigated molecular weight dependence of the overshoot recovery time.

\section{Summary}

Interrupted shear flow experiments were used to study the kinetics of the reentanglement process in concentrated solutions of high molecular weight PB. Although chain molecules are less entangled upon cessation of shearing, the time for reentanglement is longer, by ca. 1.5 decades, than the terminal relaxation time determined from linear viscoelastic measurements. Thus, even though a reduction in entanglement facilitates global chain motions, the entanglement recovery process is substantially longer than the time for reorientation of the fully entangled chain over a distance equal to its size. Despite this difference, the characteristic time for reentanglement has the same molecular weight dependence, $M^{3.2 \pm 0.4}$, as the linear viscoelastic relaxation time.

Acknowledgment. The work at NRL was supported by the Office of Naval Research. C.G.R. and S.W. thank Bridgestone Americas for permission to publish.

\section{References and Notes}

(1) Doi, M.; Edwards, S. F. The Theory of Polymer Dynamics; Clarendon: Oxford, 1986.

(2) Watanabe, H. Prog. Polym. Sci. 1999, 24, 1253

(3) Lodge, T. P.; Rotstein, N. A.; Prager, S. Adv. Chem. Phys. 1990, 79,1

(4) Doi, M. J. Polym. Sci., Polym. Phys. Ed. 1983, 21, 667.

(5) Graessley, W. W. Adv. Polym. Sci. 1982, 47, 68. 
(6) Colby, R. H.; Fetters, L. J.; Graessley, W. W. Macromolecules 1987, 20, 2226

(7) Milner, S. T.; McLeish, T. C. B. Phys. Rev. Lett. 1998, 81, 725.

(8) Marucci, G. J. Polym. Sci., Polym. Phys. Ed. 1985, 23, 159.

(9) Hua, C. C.; Kuo, H. Y. J. Polym. Sci., Polym. Phys. Ed. 2000, 38,248

(10) des Cloizeaux, J. Macromolecules 1990, 23, 4678.

(11) Wang, S. F.; von Meerwall, E. D.; Wang, S. Q.; Halasa, A.; Hsu, W. L.; Zhou, J. P.; Quirk, R. P. Macromolecules 2004 , 37,1641 .

(12) Wang, S. Q. J. Polym. Sci., Polym. Phys. Ed. 2003, 41, 1589.

(13) Schweizer, K. S. J. Chem. Phys. 1989, 91, 5802, 5822.

(14) Archer, L. A.; Mhetar, V. J. Non-Newtonian Fluid Mech. $1999,81,71$.

(15) Hua, C. C.; Schieber, J. D. J. Chem. Phys. 1998, 109, 10018.

(16) Mead, D. W.; Doi, M.; Larson, R. G. Macromoleucles 1998, $31,7895$.

(17) McLeish, T. C. B.; Larson, R. G. J. Rheol. 1998, 42, 81.

(18) Pearson, D. S.; Herbolzheimer, E.; Grizzuti, N.; Marucci, G. J. Polym. Sci., Polym. Phys. Ed. 1991, 29, 1589.

(19) Graessley, W. W. J. Chem. Phys. 1965, 43, 2696.

(20) Marucci, G. J. Non-Newtonian Fluid Mech. 1996, 60, 279

(21) Vinogradov, G. V.; Belkin, I. M. J. Polym. Sci., Part A 1965, 3,917 .

(22) Simmons, J. M. Rheol. Acta 1968, 7, 184.

(23) Tanner, R. I.; Williams, G. Rheol. Acta 1971, 10, 528.

(24) Batzer, H.; Wagner, M. H.; Meissner, J. Makromol. Chem. 1980, 181, 1533.

(25) Isono, Y.; Obashi, N.; Kase, T. Macromolecules 1995, 28, 5145.

(26) Isono, Y.; Kamohara, T.; Takano, A.; Kase, T. Rheol. Acta 1997, 35, 245.

(27) Oberhauser, J. P.; Leal, L. G.; Mead, D. W. J. Polym. Sci., Polym. Phys. Ed. 1998, 36, 265.

(28) Osaki, K.; Inoue, T.; Isomura, T. J. Polym. Sci., Polym. Phys. $E d$. 2000, 38, 2043. Osaki, K.; Inoue, T.; Uematsu, T. J. Polym. Sci., Polym. Phys. Ed. 2000, 38, 3271.

(29) Moore, J. D.; Cui, S. T.; Cochran, H. D.; Cummings, P. T. Phys. Rev. E 1999, 60, 6956.

(30) Santangelo, P. G.; Roland, C. M. J. Rheol. 2001, 45, 583.

(31) Attane, P.; Pierrard, J. M.; Turrel, G. J. Non-Newtonian Fluids 1985, 18, 295.
(32) Dealy, J. M.; Tsang, W. K.-W. J. Appl. Polym. Sci. 1981, 26, 1149.

(33) Huppler, J. D.; MacDonald, I. F.; Ashare, E.; Spriggs, T. W.; Bird, R. B.; Holmes, L. A. J. Rheol. 1967, 11, 181.

(34) Stratton, R. A.; Butcher, A. F. J. Polym. Sci., Polym. Phys. Ed. 1973, 11, 1747 .

(35) Menezes, E. V.; Graessley, W. W. J. Polym. Sci., Polym. Phys. Ed. 1982, 20, 1817.

(36) Xu, Y. Z.; Kee, D. D.; Fong, C. F. C. M. J. Appl. Polym. Sci. 1995, 55,779

(37) Bird, R. B.; Armstrong, R. C.; Hassager, O. Dynamics of Polymeric Liquids, 2nd ed.; Wiley: New York, 1987; Vol. 1.

(38) Tapadia, P.; Wang, S. Q. Phys. Rev. Lett. 2003, 91, 198301.

(39) Graessley, W. W.; Edwards, S. F. Polymer 1981, 22, 1329.

(40) Cox, W. P.; Merz, E. H. J. Polym. Sci. 1958, 28, 619.

(41) Doi, M.; Takimoto, J. Philos. Trans. R. Soc. London, Ser. A 2003, 361, 641 .

(42) Hua, C. C. J. Chem. Phys. 2000, 112, 8176.

(43) The original Cox-Merz relationship (ref 40) was developed from a comparison of $\eta^{*}(\omega)$ and shear rate dependence of capillary viscosity data. The latter were not corrected for nonNewtonian velocity profile or entrance/exit pressure effects. However, the Cox-Merz empiricism is commonly used in the literature to signify a near correspondence between $\eta^{*}$ and corrected $\eta_{\mathrm{ss}}$ when respectively plotted versus $\omega$ and true shear rate on a log-log plot.

(44) Gleissle, W. In Rheology; Astarita, G., Marrucci, G., Nicolais, L., Eds.; Plenum Press: New York, 1980; Vol. 2, p 457.

(45) Wood-Adams, P. M. J. Rheol. 2001, 45, 203.

(46) Elkissi, N.; Piau, J. M.; Attane, P.; Turrel, G. Rheol. Acta 1993, 32, 293.

(47) Osaki, K.; Watanabe, H.; Inoue, T. Nihon Reoroji Gakkaishi 1998, 26,49 .

(48) Robertson, C. G.; Roland, C. M.; Puskas, J. E. J. Rheol. 2002 , $46,307$.

(49) Berry, G. C.; Fox, T. G. Adv. Polym. Sci. 1968, 5, 261.

(50) Xu, Y. Z.; De Kee, D.; Chan, C. F. J. Appl. Polym. Sci. 1995, 55,779 .

(51) Takahashi, M.; Masuda, T.; Onogi, S. Trans. Soc. Rheol. 1977, $21,337$.

(52) Zhu, Z.; Wang, S. Q. J. Rheol. 2004, 48, 571.

MA048148G 